\title{
Le interviste INN-possibili: Aggiungere vita agli anni e non anni alla vita
}

Allungamento della vita media e della sopravvivenza in $\mathrm{CKD}$, aumento delle comorbidità, incremento della povertà, della mancanza di supporti famigliari e della carenza di risorse sociali, non sono più argomenti futuribili per il nefrologo, sono argomenti già attuali e che ci coinvolgono sempre più in una assistenza e risoluzione di problematiche di geronto-nefrologia.

Le implicazioni che ne derivano portano già a doversi interrogare su possibili diverse scelte, che oltre a tener conto di fondamentali e imprescindibili (alla nostra professione) motivi etici compendino anche trattamenti che dovranno commisurarsi con una diversa qualità di vita. Una qualità di vita attesa da una popolazione di pazienti, sempre più anziani, comorbidi, soli e bisognosi di trattamenti che non potranno alla fin fine che esser sostenibili al contesto sociale attuale e a quello previsto.

Per il nefrologo si profila una sfida epocale che potrebbe essere -per quantità e qualità- la peggiore della storia della nefrologia, una sfida che è stata perfettamente stigmatizzata da un insigne nefrologo italiano, con quello che potrebbe essere l'incipit della nefrologia dei prossimi decenni: aggiungere vita agli anni e non anni alla vita!

Poiché questo futuro è già presente e proprio perché per questa popolazione di pazienti è più importante la qualità che la quantità di vita, questa rubrica si propone di trattare l'argomento mediante il parere di esimi nefrologi e cultori a quest'argomento dedicati o quanto meno sensibili.

Una buona, ponderata e riflessiva lettura a tutti. 